https://doi.org/10.24201/aap.2021.327

INFORME

\title{
Tailandia al inicio de la década 2020
}

\section{Thailand at the Beginning of the 2020s}

\author{
JOSÉ ERNESTO RANGEL DELGADO \\ http://orcid.org/0000-0003-1392-9153 \\ Universidad de Colima, México
}

Recepción: 5 de octubre de 2020

Aceptación: 5 de enero de 2021

Resumen: El año 2020 no sólo se distingue por ser el inicio de una década, sino también por todos los retos y cambios que implica. La llegada de la covid-19 y sus repercusiones en Tailandia vinieron acompañadas por los extraordinarios reclamos populares al sistema de monarquía constitucional, así como por las decisiones tomadas por el gobierno, entre otras cuestiones. Este capítulo tiene el objetivo de presentar al país en su más reciente desenvolvimiento, de forma que se divide en una breve introducción; sus ámbitos interno y externo; su economía; su sociedad; un apartado de reflexiones finales y otro más de referencias. Así, a manera de reporte, busca matizarse con el sentido de seguridad humana que pretende el Anuario Asia Pacífico 2021 del Centro de Estudios de Asia y África de El Colegio de México.

Palabras clave: Tailandia; gobierno; política exterior; economía; sociedad; seguridad humana.

Abstract: The year 2020 is not only distinguished for being the beginning of a decade, but also for all the challenges and changes it implies. The arrival of Covid-19 and its impacts in 
Thailand were accompanied by extraordinary popular claims to the constitutional monarchy system, as well as government decisions, among other issues. The aim of this chapter is to present the Kingdom of Thailand in its most recent development. It is divided into a brief introduction, its domestic and foreign fields, its economy, its society, a section of final reflections and another one of references, which, by way of a report, seeks to be nuanced with the sense of human security that the 2021 Asia Pacific Yearbook of the Center for Asian and African Studies of El Colegio de México seeks.

Keywords: Thailand; government; foreign policy; economy; society; human security.

\section{INTRODUCCIÓN}

Tailandia, por su ubicación y política exterior, se encuentra posicionada en el Sureste Asiático como uno de los países más influyentes; es la economía número 25 a nivel global por su tamaño, con un índice de miseria económica según Bloomberg (2020) que la coloca en el último lugar entre 60 países y con un Reporte Mundial de Felicidad 2020 que la ubica en el lugar número 54 de 104 países considerados. Situación que se ha visto trastocada por los conflictos internos de un movimiento estudiantil que demanda mayor democracia, entre otros asuntos. En efecto, durante 2020 la situación económica del país se ha visto afectada por la pandemia de covid-19 asociada particularmente con la disminución del turismo, una de las ramas más importantes de su economía.

\section{ÁMBITO INTERNO}

En al ámbito interno resalta en 2020 la ola de protestas por parte de miles de jóvenes estudiantes, iniciadas a mediados del mes de julio y que se extienden a lo largo de todo el año, cobijados por la histórica contestaria Universidad de Thamasat. Este movimiento estudiantil ha incluido más de una docena de escuelas preparatorias (Wongcha-un 2020), que para evitar acusaciones de Lesa Majestad y disidencia han aludido a lord Voldemort (el que no puede ser nombrado, en la saga de Harry Potter) y el saludo de los tres dedos de los "Juegos del hambre" con el reclamo de un menor autoritarismo en las escuelas que impone 
“obediencia a la tradición, formación diaria por el himno nacional, reglas estrictas sobre uniformes, corte de cabello y conducta" (Wongcha-un 2020); reclamos a los que más tarde se sumaría el Frente por la Democracia y la Dictadura, llegando a reunir unas 200000 personas en la Plaza de la Constitución, la mayoría jóvenes, exigiendo una nueva constitución (Europapress Internacional 2020).

Sin pretender erradicarla, el movimiento estudiantil intenta además, como afirma Adrián Foncillas en un artículo de elperiódico del 22 de agosto de 2020, encorsetar la monarquía en el traje democrático: que sea fiscalizada por el parlamento, modere sus gastos, renuncie al control del ejército y no medie en la política bajo el reclamo de una verdadera democracia, esto en el marco de una competencia comercial entre potencias que fragmenta las relaciones económicas globales, enrareciendo el ambiente político y social internos del reino.

En contra de estas propuestas, el partido de derecha en el poder y cerca de 1200 personas, defensoras de la realeza Thai, la mayoría de la tercera edad, se han manifestado (30 de agosto), haciendo un llamado al respeto por la monarquía y al statu quo (Kittisilpa 2020).

Sin embargo, si bien Tailandia cuenta con un Ministerio de Desarrollo Social y Seguridad Humana, las condiciones actuales de emergencia sanitaria y la reducción de divisas producto de la disminución del turismo no le han favorecido para justificar el dato de país con menor miseria económica (Bloomberg 2020), o bien de desarrollo humano alto (PNUD 2019), o de nivel promedio en el Reporte Mundial de Felicidad (WHR 2020), y ha tratado de solventar las violaciones a los derechos humanos asociadas con el legado del mandato militar; la libertad de expresión; los arrestos militares, la tortura y las cortes militares; las desapariciones forzadas; la ausencia de información por la violencia; la defensa de los derechos humanos; la violencia y los abusos en las provincias de la frontera sur del país; los refugiados, el asilo político y los trabajadores migrantes; la igualdad de género y la influencia internacional; que no resulta ser suficiente para mejorar las condiciones de derechos humanos (Human Rights Watch 2020).

Pero el principio de la seguridad humana se encuentra altamente valorado en Tailandia, al menos en el discurso y particularmente en su relación con ASEAN, como se mencionó en el cincuenta aniversario de la organización regional: 
[...] la gente debe estar protegida y cuidada, ya que es el recurso más importante [...] La gente debería tener acceso a suficiente comida, agua y otros recursos para alcanzar una vida productiva y vivir con dignidad [...] desde la juventud hasta la fuerza laboral, desde los trabajadores emigrantes, hasta la creciente población activa envejecida [...] Asimismo, el bienestar de la gente se deberá proteger frente a los desafíos de las drogas ilegales, la trata de personas y la pandemia, entre otros. A través de la iniciativa de Tailandia, hemos avanzado en tratar de crear una ASEAN libre de drogas y el Centro ASEAN NARCO en Bangkok será una importante herramienta para ayudar a alcanzar esta meta. Tailandia también está tomando la iniciativa para mejorar el bienestar de los ancianos y darles las herramientas y las oportunidades para convertirse en una parte fundamental de la sociedad. Éste es el motivo por el que hemos lanzado la iniciativa "Envejecimiento Activo", que ayudará a mejorar la seguridad humana de nuestra población envejecida" ("El papel de Tailandia a la hora de reforzar a la ASEAN tras 50 años: Inclusividad, seguridad humana y desarrollo sostenible”, $L a$ Vanguardia, 30 de junio, 2017).

Por otro lado, la educación y la capacitación representan una tarea permanente en la agenda oficial que requieren de adaptaciones de acuerdo con las últimas manifestaciones estudiantiles, particularmente en cuanto a un sistema educativo centrado en valores democráticos, a decir de los estudiantes responsables de las últimas manifestaciones (Vejpongsa 2020); así como la consideración de aspectos como el regional (Tailandia se divide en 77 provincias); el financiamiento de la educación que por más elevado no logra resultados trascendentes; la pobreza y la equidad; las características de lo público y lo privado en la educación que decantan un alto costo de la educación; el desempeño docente que asume las condiciones del gobierno en beneficio de sus propios intereses como gremio; la calidad y la importancia del enfoque de competencias como una estrategia que permite una mayor participación del estudiante en su propio proceso educativo.

Todo lo anterior tiene un importante impacto en el mercado laboral de egresados, el cual se enfrenta a una industria de cuarta generación (i4.0) centrada en la consolidación de conocimientos que a su vez exhibe el gran trabajo pendiente en el sector educativo. Esta carencia se refleja en la inconformidad mostrada por estudiantes y algunos sectores de la sociedad que se manifiestan por una mayor democratización de los procesos internos del gobierno, mayor libertad y protección por abusos de las personas (Prachatai 2020). 


\section{ÁMBITO EXTERIOR}

Quienes protestan no parecen estar solos en la región; a sus reclamos se han unido movimientos de jóvenes sin fronteras por la democracia en Taiwán y Hong Kong por medio de la así llamada "Alianza del té con leche" (que hace referencia al gusto que comparten por el té dulce). En el fondo, esa alianza representa una rebelión contra regímenes que se enfrentan a reclamos en favor de una mayor democracia, como es el caso de Rusia, por el supuesto envenenamiento del líder opositor al Kremlin, Alexey Nalvalny, apoyado por Alemania; o Filipinas, la India o Singapur, que han mostrado interés en la "Alianza del té con leche" en la base de las disputas con China por ciertas islas del Mar Meridional y las revueltas en Hong Kong, o por las escaramuzas recientes en la frontera con la India o por el tema pendiente de la represión del activista Roy Ngerng en contra del primer ministro de Singapur, Lee Hsien, en 2014. En todo caso, parece ser cierto que la "solidaridad está tomando una forma más organizada y estructural" (Tanakasempipat y Chow 2020).

Lo anterior se suma al hecho de que la política exterior de Tailandia se ha caracterizado por ser equilibrada y omnidireccional, matizada de regionalismo, de coexistencia pacífica y de buenas relaciones con los países de la ASEAN, así como por su flexibilidad y pragmatismo bajo un discurso de paz, estabilidad y seguridad cooperativa y multilateral en el Sudeste Asiático y con las principales potencias.

No obstante, un histórico sentimiento antichino y la creciente desconfianza en Estados Unidos en materia de seguridad propician que la ASEAN preste atención por un lado a la cercanía de Tailandia con China, colocándola como intermediaria, pero también al fortalecimiento de la ASEAN con otros socios, para reducir al mínimo las preocupaciones que le causan las tensiones entre Estados Unidos y China (Rakhmat 2020).

Es así como Tailandia da preferencia a la relación con sus vecinos (ASEAN+6 - China, Japón, Corea del Sur, India, Australia y Nueva Zelanda-) y a su relación bilateral con Japón. Tailandia no tiene conflictos territoriales con China, y sus relaciones se han intensificado después del golpe de Estado de 2014, conformándose en una suerte de contrapeso a la presión ejercida por Estados Unidos, como la suspensión de beneficios preferenciales al comercio de 231 productos tailandeses que, si bien no fue recibida por el gobierno de Tailandia como una 
decisión que afectará sus exportaciones totales a Estados Unidos, es posible visualizarla precisamente por el acercamiento de Tailandia a China (Arunmas 2020a); sumado al apoyo al movimiento estudiantil por parte de Estados Unidos (Shim 2020).

Es así como en 2020 Tailandia pone en tercer lugar su relación con Estados Unidos, que fuera su principal aliado después de la Segunda Guerra Mundial para la contención del comunismo en el Sudeste Asiático; y coloca en cuarto lugar a la Unión Europea, particularmente por el interés de intensificar sus relaciones comerciales, proceso que se ha ralentizado después del golpe de 2014.

Ciertamente, otro de los temas de gran interés en el ámbito externo resulta ser el de la seguridad humana por el tráfico de drogas, la migración y la utilización del agua que proporcionan el Río Mekong y sus afluentes.

De forma que sus relaciones exteriores se han caracterizado — con Myanmar, Laos, Camboya y Malasia - por el tráfico de drogas y la migración irregular de trabajadores procedentes de Myanmar y de miles de desplazados (100 000) que se concentran en campos de refugiados en sus fronteras; así como en sus relaciones con Malasia por los problemas separatistas de las provincias del sur de Tailandia, donde existe una gran comunidad musulmana de origen malayo, y con Camboya por los problemas de delimitación de fronteras por el templo hinduista de Preah Vihear, problema que ya parece haberse resuelto dando lugar a una nueva era de relaciones bilaterales enfocadas en la cooperación para el desarrollo económico de acuerdo con la ASEAN, pilar de la política exterior tailandesa (National Identity Board 2000).

Asociado a los problemas de seguridad humana se encuentra el de la seguridad alimentaria, con aristas muy bien identificadas en el ámbito internacional, particularmente en cuanto al control del agua y sus impactos en la producción de alimentos. De tal forma que, entre las causas de grandes disputas internacionales, se han detectado para el futuro las relacionadas con la disponibilidad de agua por las naciones en el mundo. En este sentido, un asunto de mayor importancia para la política exterior de Tailandia es la disponibilidad de agua en el bajo Mekong, y su relación con sus vecinos, particularmente con China. Para desahogar este problema y articular las relaciones entre todos los países involucrados se han creado la Comisión del Río Mekong (con sede en Beijing) y el Grupo para la Cooperación de los ríos Lankang-Mekong. 
Una investigación realizada por una compañía estadunidense encontró que en 2019 el control ejercido por China en el alto Mekong durante la sequía de ese año afectó la vida de pescadores y granjeros de Laos, Tailandia, Myanmar, Camboya y Vietnam, lo que Beijing desconoce (Wongcha-un y Crossley 2020). De esta forma se ha generado una disputa entre naciones por la disponibilidad del agua de los afluentes, la cual expone con claridad que toda acción de control del agua en el alto Mekong implica una reacción en el bajo Mekong que recorre la península, por lo que compartir información al respecto resulta de la mayor importancia para el diseño de la política pública doméstica de cada uno de los países involucrados.

Al inicio de la década de 2020, la situación en materia de política exterior no ha cambiado en lo sustantivo. Sin embargo, la pandemia de covid-19 ha contribuido más en la orientación de algunos aspectos que se suman a los ya comentados y que a partir de la información disponible nos han parecido representativos por que se encuentran asociados al deterioro de la seguridad humana: aquellos que se relacionan con el desempleo de migrantes propiciado por la pandemia; la violencia política que ha desencadenado la manifestación de miles de estudiantes que reclaman mejores condiciones de democracia; la condición de los apátridas que llega ya al medio millón de individuos; de los refugiados que permanecen en los campos de concentración de sus fronteras; la lucha antidrogas; la repatriación propiciada por la pandemia; el cierre de acceso a los extranjeros al país y la importación de armamento desde China.

La Organización Internacional del Trabajo (Engblon et al. 2020), por ejemplo, considera que los trabajadores migrantes se encuentran entre los grupos más vulnerables durante la pandemia de covid-19, por lo que sugiere extender las fechas límites para la renovación de permisos de trabajo y de visas; desarrollar líneas de acción y requerimientos mínimos de hospedaje y dormitorio a los trabajadores migrantes; prevenir y combatir la propagación de covid-19 en los centros de detención de migrantes; asegurar que los trabajadores migrantes desempleados tengan acceso a esquemas de desempleo y otros beneficios de protección social; exención de pago de visa incluyendo los pagos para visa de retorno de trabajadores migrantes; desagregar las estadísticas según el género; fortalecer las campañas de información pública orientadas a los trabajadores migrantes e incluir 
información sobre las necesidades específicas de las mujeres; asegurar que los empleadores de trabajadores migrantes mitiguen todos los riesgos que impliquen exponerse al incremento de covid-19; desarrollar lineamientos de salud y seguridad ocupacional; asegurar que los despidos de los trabajadores no conlleven un sentido discriminatorio; asegurar que quien perdió su empleo reciba los salarios, la seguridad social y otros beneficios así como su documentación correspondiente; asegurar que todos los trabajadores migrantes tengan acceso a atención legal y compensación por trato injusto; asegurar el acceso gratuito a pruebas de laboratorio por covid-19, facilidades de cuarentena y permisos de distanciamiento social; protección social y medidas de estímulo para enfrentar los impactos económicos de la pandemia; alimentación, abrigo y provisión de equipo como gel desinfectante y cubrebocas; asegurarse de liberar a todos los inmigrantes detenidos bajo condiciones insalubres; coordinarse con los países de origen de los trabajadores migrantes para su retorno.

Un aspecto adicional que es posible comentar en materia de seguridad humana, asociado con el ámbito exterior de Tailandia, es la situación en que se encuentra medio millón de apátridas, producto de las lagunas normativas en este aspecto y de la situación irregular en que se hallan estas personas que, habiendo nacido en el país, no cuentan con las condiciones mínimas de protección a la salud y otras garantías de un ciudadano común, con lo que en situación de pandemia y de pobreza, que se incrementa por falta de empleo, se complican aún más (Ruiz-Canela 2020). A este problema se suma el de los refugiados que prevalecen en los campos de concentración en sus fronteras, particularmente con Myanmar, donde las condiciones extremas prevalecen a pesar de los esfuerzos por parte del gobierno de Tailandia y de donantes como Italia, Japón, República de Corea, España, Suecia y Estados Unidos. Según Naciones Unidas, la cifra se acerca a 95000 personas refugiadas en diversos puntos a lo largo de la frontera de Tailandia con Myanmar, concentrándose en el rango de 18 a 59 años de edad, con $27.5 \%$ de mujeres y $26.0 \%$ de hombres, provenientes en $84 \%$ de la etnia Karen y 68\% de la región de Kayin (UNHCR Office 2020).

La lucha antidrogas prevalece, de forma que de abril a junio de 2020 las autoridades arrestaron a un total de 1374 narcotraficantes y confiscaron un volumen de drogas, mayormente metanfetaminas, valorado en \$17.1 millones de dólares durante una campaña en la región norte del país según la Administración de Drogas; mientras que en la provincia 
sureña de Surat Thani, en el mes de julio se incautaron 1200 kilogramos de metanfetaminas provenientes de Myanmar y con destino a Malasia, Indonesia, Filipinas, Japón y Australia (VietnamPlus 2020a).

Asimismo, la repatriación de extranjeros — principalmente hacia Vietnam y Malasia (VietnamPlus 2020b; Malay Mail 2020a) — se agudiza por la emergencia de salud y a partir de que Tailandia decidiera cerrar el acceso temporal a extranjeros, permitiendo en una primera etapa el acceso sólo a quienes tienen familia en Tailandia, vínculos laborales con el país, estudiantes, expertos técnicos de negocios, inversionistas, e invitados especiales provenientes de todas partes (AP News 2020), principalmente de Myanmar y Malasia (Spanish.xinhua.com 2020). Posteriormente, el primer ministro Prayut Chan-o-cha ha instado en el marco de la ASEAN a colaborar en materia de salud pública para la reapertura de las fronteras intrarregionales, con la finalidad de restaurar la economía regional, por lo que se tiene la tarea de recuperar poco a poco las condiciones económicas perdidas en materia de turismo por el cierre de sus fronteras (Bangkok Post 2020a), a lo cual se ha sumado Singapur para facilitar los viajes esenciales de negocios (Fan 2020).

Otro asunto que caracteriza las condiciones de Tailandia con el exterior es el de las disputas comerciales con Filipinas por su exportación de tabaco, que ha merecido la intervención de la OMC.

Filipinas está tratando de obtener la aprobación de la OMC para suspender las concesiones sobre las importaciones tailandesas de 594 millones de dólares como represalia por el incumplimiento por parte de Tailandia del fallo sobre su controversia relativa a los cigarrillos [...] por su parte, Bangkok argumenta que su apelación está pendiente de revisión [...] y que los procedimientos tendrían que detenerse mientras que el Órgano de Apelación se completa con un quórum de tres jueces (Rosales 2020; Tobacco Reporter 2020).

De igual manera, destacan aspectos en el ámbito exterior debido al crecimiento del comercio con Camboya, que continúa a pesar de la pandemia y que, aun bajo las condiciones extremas de emergencia, sigue creciendo, de forma que en el primer semestre de este año se ubicó en \$2 600 millones de dólares, lo cual muestra un crecimiento de 2.27\%, a diferencia de la disminución de su comercio con otros países vecinos ocasionada en gran parte por la propagación de la covid-19 (Kunmakara 2020). Sobresale también el asunto del Sistema de 
Cable MIST, bajo los auspicios de Orient Link (OLL) y NEC Corp (NEC), para una conexión digital que busca favorecer la conectividad entre Singapur, Malasia, Myanmar, la India y la propia Tailandia, y que pretende ser concluida hacia fines de 2022, haciendo uso de la 5G para SNS, y los servicios de nube en beneficio de negocios y sociedad en su conjunto (Japan Today, NEC 2020). Del mismo modo destaca el tema de la importación de armamento desde China, como es la compra de armamento y de tres submarinos que, a la fecha de este reporte, se había retrasado por los reclamos de la sociedad tailandesa y de grupos opositores al gobierno, lo que muestra un ejemplo más del manejo de la política exterior por parte del gobierno (Reuters 2020).

Tailandia, como muchos países más, ha tenido que reagrupar sus preferencias de política exterior tratando de respetar su filosofía primaria que la sustenta al menor costo posible. Producto de condiciones políticas internas, destaca su acercamiento con China y su aparente alejamiento de Estados Unidos, a partir del golpe de Estado de 2014, que sumado a una situación preocupante en torno a su monarquía, así como por la postura de un gobierno interesado en mantener la situación en orden, provocan un estrés en la sociedad, exacerbada por la situación de emergencia de salud que atraviesa el país. Todo lo anterior ha impactado en su política doméstica, en su economía, su sociedad y en el manejo de una política exterior sui generis.

\section{ECONOMÍA}

La crisis económica por la que Tailandia está atravesando en 2020, producto de la pandemia ocasionada por el virus SARS-CoV-2, ha impactado desde fuera a diferencia de la crisis de 1997. En la actualidad, la crisis se atribuye a políticas de gobierno erráticas o arriesgadas que afectan todos los sectores de la economía, principalmente el turismo, del cual Tailandia es altamente dependiente y que representa $15 \%$ del producto interno bruto (The World Bank 2020). De forma que el cierre de fronteras generó una disminución de turistas de $60 \%$, reduciendo sustancialmente los ingresos por el concepto (MarketScreener 2020).

Sumado a lo anterior, se prevé que la economía tailandesa se contraiga 5\% en 2020 (empezando a recuperarse apenas en 2021 con una contracción de $4.1 \%$ y 3.6\% en 2022), lo que constituye una de las disminuciones más notorias previstas en la región de Asia Oriental 
y el Pacífico, propiciadas por el debilitamiento de la demanda mundial con la consecuente reducción de las exportaciones de Tailandia en 23\% en junio, principalmente de automóviles (70.1\%), arroz (25\%) y caucho (55.6\%), según datos del Ministerio de Comercio (EFE 2020); la ralentización de cadenas de valor mundiales, como la del automóvil; las restricciones a la movilidad que han afectado el consumo y las ventas de bienes duraderos en casi $12 \%$ durante el primer trimestre de 2020; la caída de la demanda y los precios de la energía con graves pérdidas de empleo; todo lo cual hace prever una recuperación económica prolongada e incierta (The World Bank 2020).

Ante esta situación el gobierno ha aplicado paquetes de respuesta que ascienden a $12.9 \%$ del PIB, particularmente para atender a las personas más vulnerables que viven con menos de $\$ 5.5$ dólares al día, y que se prevé se duplicarán, pasando de 4.7 millones en el primer trimestre de 2020 a unos 9.7 millones en el segundo, antes de recuperarse ligeramente hasta los 7.8 millones en el tercer trimestre del mismo año. Entre estas medidas destacan: $a$ ) la transferencia de efectivo a los hogares, favoreciendo el consumo con un gasto de \$1 400 millones de dólares estadunidenses distribuidos entre 15 millones de personas afectadas (VietnamPlus 2020c) y los proyectos de infraestructura de la economía local (5.9\% del PIB) que se financiarán parcialmente mediante préstamos; $b$ ) el fondo de estabilización del mercado de bonos corporativos creado por el Banco de Tailandia, para ayudar a las empresas a renovar los bonos con vencimiento (2.4\% del PIB) y financiar préstamos en condiciones favorables a las PyMES $(2.9 \%$ del PIB $) ; c)$ la reducción de impuestos que requerirá de una reconstrucción de las reservas fiscales a largo plazo para optimizarse hacia crisis futuras e invertir de manera planificada en infraestructura pública; $d$ ) la reestructuración de la deuda de las empresas y los hogares. Éstas, sin embargo, habrán de enfrentarse al problema de insuficiente conectividad de los más vulnerables para obtener los beneficios que el gobierno pretende otorgarles, detectar las deficiencias y diseñar políticas públicas ad hoc que puedan adaptarse a los distintos sectores de la economía y a un mercado laboral que requiere de capacitación de acuerdo con las condiciones cambiantes del entorno sin dejar de considerar habilidades socioemocionales, cognitivas y técnicas de orden superior (The World Bank 2020). 
Aparte de lo anterior, se esperan grandes tareas como la modernización de la agricultura para asegurar la producción de alimentos haciendo uso de la biotecnología y la gestión de la ciencia y la innovación asociadas con el bienestar; la inversión extranjera directa y la importación de bienes y servicios necesarios para complementar el sano funcionamiento del mercado, sea de oferta (empresa/industria) o de demanda (comercio/consumo); así como el posicionamiento en el mercado externo de la producción interna manifiesto en el comportamiento de sus exportaciones para la generación de divisas, como puede ser el caso de la renovación de la industria automotriz con la producción de los "autos verdes" para mejorar las condiciones de contaminación del aire, con el plan de llegar a 30\% de la producción total de vehículos eléctricos para el 2030 (VietnamPlus 2020d); la apropiada gestión de las finanzas y de los impuestos para evitar desbalances ante eventuales crisis como la ocasionada por la actual pandemia; la inversión en infraestructura orientada a mejorar las condiciones de vida a partir de los sectores salud, transporte y comunicación, como pudiera ser el caso del impacto del turismo médico en el mejoramiento del sistema de salud tailandés, a propósito de la pandemia y de los buenos resultados que Tailandia ha tenido para enfrentarla, la renovación de su sistema ferroviario — hub, ferroviario de Ayutthaya y la red ferroviaria urbana de Bangkok-, así como la incorporación de redes de comunicación 5G (Alconchel 2011; Brown 2020; VietnamPlus 2020e); la atención del medio ambiente vinculado con los ODS de Naciones Unidas ajustados en tiempo y espacio a las condiciones internas del país, particularmente en el ámbito del gran sector del turismo (sobre el cual ya se ha comentado), pero también de la industria restaurantera afectada por covid-19 (The Nation Thailand 2020a); todos ellos con un mercado de trabajo que pretende funcionar en mejores condiciones laborales y de seguridad de los individuos involucrados.

Respecto a la agricultura, por ejemplo, Tailandia trabaja en su modernización haciendo uso de avances tecnológicos con una base bioeconómica que forma parte de las diez industrias de tipo "curva-S", cuyo objetivo general es mejorar la economía nacional a partir de la ciencia, la tecnología y la innovación (i4.0), para promover así el uso de recursos renovables en una suerte de "economía circular" como alternativa a una "economía lineal" que genera grandes cantidades de desechos orgánicos sin aprovechar. 
A decir de expertos, por medio de la biotecnología cultivos como la caña de azúcar, la mandioca, el arroz y otros, pueden ser convertidos en productos de alto valor agregado teniendo el cuidado de no generar una producción genéticamente modificada que pueda dañar la salud humana, sobre todo porque el gobierno tiene proyectada para 2026 la estrategia de transformar por esta vía el sector de los alimentos para seres humanos y animales - además de la bioenergía, bioquímicos y los biofarmacéuticos-(VietnamPlus 2020f), lo que resulta en una suerte de alternativa deseable ante el riesgo severo de una escasez de agua al que se enfrenta Tailandia, principalmente debido a la brevedad de la temporada de lluvias (VietnamPlus 2020g), y los problemas que implica la distribución de los afluentes del Río Mekong frente a las sequías de temporada, como ya se comentó.

Las importaciones de bienes y servicios, el comercio transfronterizo - incluido el de tránsito que se ha visto disminuido en poco más de \$20000 millones de dólares estadunidenses debido al cierre de los puntos de control fronterizo (Malay Mail 2020b)_, tanto como la inversión proveniente del extranjero, representan tres segmentos del ámbito económico sumamente importantes, sea por ventajas comparativas o por ventajas competitivas o por las cadenas de valor en las cuales se encuentra inserto el país; más aún por las tensiones comerciales entre China y Estados Unidos e incluso Japón, potencias con las que Tailandia tiene una estrecha cercanía económica.

Al respecto, por ejemplo, se puede mencionar la compra de 72 trenes con un total de 288 vagones de tecnología no tripulada, totalmente automáticos comprados a China, cuya entrega de los dos primeros trenes se dio en el mes de septiembre 2020, para ser utilizados en 2022 en dos líneas de tránsito ferroviario en la zona urbana de Bangkok con la intermediación de la compañía CRRC Puzhen Bombardier Transportation Systems (HEFEI, Xinhua 2020), o la "apertura en julio pasado de 2020 de la segunda tienda minorista Apple Store", según anunció en su blog ipadizate.es; así como el memorándum de entendimiento para la transferencia tecnológica desde las regiones Shimane y Osaka en Japón, la primera especializada en avance tecnológico en la agricultura, y la segunda líder en industria electrónica, ambas útiles para favorecer las industrias de autopartes, de alimentos, equipos médicos y cosméticos, esto es, segmentos de la economía que se han desatendido debido a la situación comercial entre China y Estados Unidos (The Nation Thailand 2020b). 
Sin embargo, en julio de 2020, el cierre de empresas se ha incrementado $38.4 \%$. El tipo de empresa más afectado se encuentra principalmente en la hotelería y negocios relacionados con el turismo, así como del ramo textil y del sector automotriz, en total 13400 en lo que va del año (Somruedi Banchongduang 2020).

De forma que el ambiente de negocios resulta ser uno de los aspectos más importantes para el ejercicio del mercado y la competitividad, ya que la mejora en el mismo ayuda a la recuperación económica con mayor velocidad. Al respecto, el gobierno tiene una gran tarea que realizar, particularmente proporcionando préstamos blandos a las pequeñas y medianas empresas, y promoviendo el gasto interno específicamente en el sector turístico.

Si bien es cierto que la percepción de las empresas en relación con los negocios ha aumentado, producto del relajamiento en el cierre de las fronteras, la intranquilidad por el resurgimiento de un segundo brote del nuevo coronavirus así como por las perspectivas económicas, el empleo, el comercio, la inversión y la situación política, continúan siendo una gran preocupación según el último informe del Consejo Nacional de Desarrollo Económico y Social (NESDC, por sus siglas en inglés), que recientemente informó que la contracción del PIB ha representado la más elevada desde la crisis financiera asiática de 1997-1998, reducción atribuida en gran parte a la debilidad de la exportación de bienes y servicios; la inversión y el consumo privado; la fuerte disminución del número de turistas extranjeros y los ingresos generados por ellos; la grave recesión económica mundial y la subsiguiente caída del comercio de mercancías; el impacto de la pandemia, y las condiciones de sequía, que han obligado al Consejo a reducir el pronóstico del crecimiento del PIB (Arunmas 2020b).

Con la finalidad de proporcionar mayor tranquilidad al ambiente de negocios, el primer ministro Prayut Chan-o-cha se reunió con los líderes del sector inmobiliario, dejando claro que a pesar de la crisis los compradores domésticos y extranjeros aún se encuentran interesados en la adquisición de propiedades en Tailandia; más aún, el gobierno se ha comprometido en generar paquetes económicos especiales para la compra de bienes inmobiliarios, lo que ha dado una señal de confianza para la restauración del sector de la construcción, un sector clave para la generación de empleo y la movilización del mercado interno (The Nation Thailand 2020c). 
La pandemia está marcando la economía con el cierre de negocios y el desempleo. Respecto a este último, y dado un mercado laboral vulnerable, el número de trabajadores subempleados y con permiso de trabajo podría alcanzar tres a cinco millones en el tercer trimestre del año. En el segundo trimestre, el número de trabajadores desempleados de Tailandia ascendió a un total de 745 000, lo que supone un aumento de casi dos dígitos en comparación con el periodo correspondiente a 2019. La tasa de desempleo del país aumentó a $1.95 \%$ en el trimestre de abril a junio, un récord desde el primer trimestre de 2009 (Somruedi Banchongduang 2020).

El sector de los servicios, hoteles y restaurantes fue el que más desempleo experimentó, ya que se ha visto afectado por el brote, en particular las empresas que dependen de la demanda externa y de los turistas extranjeros. El número de personas desempleadas en los negocios de hospedaje y restaurantes fue de alrededor de 84000 en el segundo trimestre, mientras que en otros servicios fue de 91000 . Al mismo tiempo, los hoteles y restaurantes mostraron la tasa de desempleo más alta, con $2.9 \%$, seguido por $2.8 \%$ de la construcción, y la electrónica con $2 \%$. Los desempleados de 15 a 24 años de edad están compuestos en su mayoría por graduados. Los jóvenes egresados integran un segmento de riesgo clave que muestra un desempleo de 324000 personas, equivalente a 98.2\% del desempleo total en el segundo trimestre, frente a los 209000 registrados en el primero, de forma que la tasa de desempleo juvenil en el segundo trimestre fue de 8.6\% (Somruedi Banchongduang 2020).

Asimismo, el subempleo también aumentó como resultado de la reducción de horas tanto para los trabajadores a tiempo completo como para los que trabajan horas extras, razón por la que las horas de trabajo por semana disminuyeron a 38.1 en promedio en el segundo trimestre, por debajo de las 40.7 del primer trimestre y las 42.7 horas de finales de 2019 (Somruedi Banchongduang 2020).

Debido a lo anterior, la cultura del trabajo está cambiando e impactando en la salud y el bienestar de las personas. Al respecto, un estudio realizado por Cigna Corporation (compañía de servicios de salud global dedicada a mejorar la salud, el bienestar y la tranquilidad de las personas a las que da servicio en diferentes partes del mundo), ha mostrado a Tailandia como un país con un mayor número de personas que en el futuro querría seguir trabajando desde casa al menos la mitad del tiempo; así como también entre los países 
que en menor medida desearían volver a la oficina de tiempo completo, pero también entre los más preocupados por los protocolos de seguridad como el distanciamiento social y el uso de mascarillas (CISION PR Newswire 2020).

Al mismo tiempo se ha ido incrementando la exportación de mano de obra a otros países, dado que Tailandia está siendo considerada internacionalmente por su disciplina y habilidades, como por ser una fuente confiable de mano de obra libre de covid-19. A Taiwán, por ejemplo, envía 20120 trabajadores con la finalidad de generar remesas que estimulen la economía doméstica. Otros países a los que la mano de obra Thai está dispuesta a trasladarse por empleo son Japón, Corea del Sur, Malasia, Singapur e Israel (Bangkok Post 2020b), lo cual requerirá asegurar la protección laboral y la seguridad humana de las personas en su condición de extranjeros.

\section{SOCIEDAD}

Como en cualquiera de los apartados aquí tratados, la complejidad resulta mayor. De acuerdo con su cultura y tradición, la sociedad tailandesa se mueve entre los valores humanos, religiosos y civiles. Además representa un espacio en el que se despliega un mosaico intercultural que le imprime diversidad y orgullo. Quizá por eso su grado de tolerancia es notorio en muchos sentidos, sumado a que Tailandia ha llegado a posicionarse entre los países con mayor apertura del Sureste de Asia, lo que la hace más dependiente del exterior teniendo que enfrentar duramente a su yo interior. De ahí que se hayan soportado tantos golpes de Estado (de 16 intentos, 12 han resultado exitosos, desde el fin de la monarquía absoluta en 1932) (Camroux 2014) en una suerte de tsunamis políticos que retraen restricción de libertades y desapariciones forzadas, como ha sido el caso del activista por los derechos humanos en Tailandia y por la protección del VIH en varios países Wanchalearm Satsaksit, quien en un exilio voluntario a Camboya se encuentra desaparecido, o el de otros ocho activistas contrarios a la monarquía y el régimen (The Nation Thailand 2020d; Beech 2020; Bangkok Post 2020c). Todo lo cual contradictoriamente se ve matizado de flexibilidad en una maravilla tropical del turismo, que muchas veces conduce a altos grados de corrupción. De acuerdo con el Índice de Percepción de Corrupción, por ejemplo, Tailandia se ubica en el lugar 104, ocupando el sexto lugar en ASEAN (Transparencia Internacional 2019), a lo que se 
agrega la humillación de la sociedad por el caso del heredero de Red Bull, quien atropelló a un policía años atrás y que aún elude la justicia, haciendo alarde, a decir de muchos, de un supuesto tráfico de influencias que ha causado un alto grado de inconformidad, y mostrando un gran reto de justicia y respeto a la legalidad, que afecta la confianza de la gente a todo el gobierno (Bangkok Post 2020d).

En 2020 minorías como el caso LGBT+ recientemente han visto una mejora en sus condiciones sociales, por una propuesta de ley por parte del gobierno para uniones civiles de parejas del mismo sexo (Ramos 2020) y otros grupos que conforman la sociedad, lo cual representa una lucha del presente con un pasado de cultura y tradición, como es posible constatar en el golpe de Estado de 2014 y las elecciones posteriores que abrieron espacio a la junta militar en el poder para legitimarse como gobierno civil; sumando, además, la necesidad del país de seguir convirtiéndose en una sociedad cada vez más moderna de acuerdo con las tendencias globales. Por su complejidad, esta lucha no se ha dado sin costos, agravados ahora por la actual situación propiciada por la pandemia de SARS-CoV-2 y cuyos impactos en la economía requieren de un gobierno atento a las condiciones internas, pero también a las externas que le rodean en su contexto particularmente inmediato, como es el caso de la ASEAN y otras organizaciones internacionales vinculadas con la región del Sureste de Asia para la toma de decisiones, muchas veces en contra de su tradición, cultura y orgullo, con un impacto notorio en su estructura social.

Actualmente, la lucha por la democracia se encuentra enarbolada por el sector educativo. Una vez más las manifestaciones de inconformidad vienen de instituciones del ramo, que reclaman una aplicación definitiva de la última reforma educativa (1999), calificada por políticos y expertos como en "proceso" para la transformación de dicho sector, que va desde la transformación de la enseñanza/aprendizaje en las aulas, hasta la uniformidad de la vestimenta diaria de los alumnos, quienes se ven obligados a portar un uniforme semejando una organización castrense, con muchas escuelas que reclaman mayores espacios de libertad, como ejemplo. Ya se ha comentado aquí al respecto, pero también se sugiere consultar sobre estos y otros temas de la educación en Tailandia el excelente libro de Fry (2018), Education in Thailand An Old Elephant in Search of a New Mahout. 
Por otro lado, pero en el mismo contexto, la situación en que se encuentra la lucha entre religiones dentro del país marca la sociedad tailandesa. Si bien actualmente (2020) el gobierno continúa trabajando de manera activa por el respeto a las minorías musulmanas de las provincias del sur, ligadas más a la tradición malaya, el proceso de pacificación se encuentra alterado a partir de los movimientos estudiantiles recientes. La comunidad musulmana continúa sintiéndose excluida ante la influencia del idioma Thai y del budismo imperante en todo el país. A lo que se añaden los diversos campos de refugiados a lo largo de la frontera con Myanmar y el medio millón de apátridas que reportan las Naciones Unidas, generando grandes problemas que caracterizan a la sociedad tailandesa, cuya economía se soporta en un mercado de trabajo con alto número de migrantes provenientes de países vecinos, que sin conocer el idioma se enfrentan a una normatividad que muchas veces no alcanzan a comprender en su totalidad, propiciándoles quedar en una situación ilegal, cuya línea divisoria sutil con frecuencia se transforma en discriminación e inseguridad humana.

En esta misma línea de la complejidad social, el número de suicidios se ha incrementado en 22\%; 2551 en los primeros seis meses del año, comparado con el mismo periodo de 2019, cuando se quitaron la vida 2092 personas, con un total de 4419 víctimas, es decir, seis de cada 100000 habitantes, debido a relaciones personales, enfermedad, alcoholismo y pobreza. Dicho incremento se agudiza en el contexto de la actual situación de pandemia, ante lo cual el gobierno ha puesto a disposición de la población la línea 1323 y ha organizado una campaña de prevención con apoyo de influencers en redes sociales, de acuerdo con el departamento de Salud Mental (Bangkok Post, Post Reporters 2020a; Salvá 2020).

A propósito del derecho humano a la vivienda y al problema de la invasión de tierras estatales por residentes de la localidad de Khon Kaen manifestado en 2020, el Departamento del Tesoro, con base en el proyecto Thanarak Pracharath, ha entregado contratos de arrendamiento a 1100 personas en el distrito de Ban Hat y tiene planes de otorgar títulos de propiedad a 73427 personas, cubriendo una totalidad de 1.05 millones de rai, medida equivalente a 1600 metros cuadrados de tierra (Bangkok Post, Post Reporters 2020b). El problema de los "sin tierra" conlleva el derecho a la vivienda como un aspecto más de la seguridad humana que debe atenderse y que se presenta como un problema social que 
prevalece en Tailandia y que no pasa inadvertido aun en momentos de pandemia, ya que el gobierno ha exentado por un año del pago de la renta por tal motivo a los involucrados. Este derecho de propiedad de la tierra también se extiende al uso del suelo para la producción agrícola, pero que implica el pago de una renta adicional.

Asimismo, Tailandia no resulta la excepción respecto al envejecimiento de su población, lo que muchas veces explica un mercado laboral matizado de migrantes ilegales. De forma que se espera que aproximadamente $23 \%$ de la población alcance una edad mayor de 65 años para 2035, lo que representará un gasto de 1.4 billones de Bahts (44 800 millones de dólares estadunidenses), y se agudizará aún más, según prevé el Fondo Monetario Internacional, ya que la tasa de dependencia de personas mayores sobre la población en edad de trabajar (lo cual es usual por tradición en los hogares asiáticos) llegará hasta 50\% para el 2050; situación que en efecto se encuentra estrechamente relacionada con la seguridad humana y de la cual no es posible escindirse, pues es una realidad para el caso de Tailandia, cuyo sistema de vida familiar se constituye de hasta cuatro generaciones (The ASEAN Post 2020) y eso enfrenta a los gobiernos a medidas de bienestar social pero también a las familias a actitudes de solidaridad con sus adultos mayores.

En el marco de esta complejidad, el tejido social de Tailandia podría estar desarticulándose debido al acontecer global al que se encuentra expuesto con impacto, a su vez, en la seguridad humana. Un ejemplo es la matanza sin precedentes - emulando eventos similares en otras sociedades, casi de ficción, pero sucedida realmente- en un centro comercial de Bangkok propiciada a principios de año por un miembro del ejército. Si bien Tailandia es uno de los países con mayor número de armas en el mundo, con más de 10 millones pertenecientes a civiles, entre legales e ilegales con una media de 15 por cada 100 personas (Karp 2018), es inusual una matanza de esta naturaleza, ya que los asaltos con armas de fuego no son habituales en el país (Caballero 2020).

\section{REFLEXIONES FINALES}

Como una ruta de trabajo, en el presente reporte se considera la seguridad humana en Tailandia, esto es, la ampliación de las capacidades, las libertades y la protección por abusos 
a las personas. Este mejoramiento se bifurca entre el deber ser y la práctica tanto en el ámbito político en su condición interna y externa, como en el económico y social.

Este esfuerzo de articulación ha considerado aspectos como la democracia, la libertad de expresión, el respeto, la tolerancia, y el derecho a la educación y a la capacitación en el trabajo, que se pretende dejar patentes como un indicador de algunas de las aristas asociadas con la seguridad humana a la que se refieren Font y Ortega (2012).

Es que ante procesos de globalización envolventes como los que las sociedades han tenido que enfrentar, las estructuras sociales internas en muchos espacios del mundo están debilitándose. En medio de las tensiones comerciales entre potencias, el tráfico de drogas y de armas, la lucha contra el terrorismo, pandemias como la covid-19, pero también la conformación de un nuevo orden mundial que se construye en una lucha por una hegemonía compartida, Tailandia participa activamente de manera abierta y decidida, pero con altos costos.

José Ernesto Rangel Delgado tiene doctorado con especialidad en Asia, África y América Latina, por la Academia de Ciencias de Rusia. PTC de la Universidad de Colima. Pertenece a PRODEP-SEP, SNI-Conacyt, y cultiva la línea de investigación sobre Relaciones Económicas e Internacionales en la Cuenca del Pacífico. Ha dirigido/codirigido y sido sinodal de tesis de licenciatura y posgrado; participado en eventos nacionales e internacionales sobre temas de Asia Pacífico; autor y coautor de artículos, libros y capítulos de libros, entre los que se encuentra Educación superior y empleo en dos economías de la Cuenca del Pacifico. Casos México y Tailandia, publicado por la Universidad de Colima y el Pacific Circle Consortium (2008); su versión ampliada es The crisis in Two Pacific Rim Economies. Higher Education and Employment in Mexico and Thailand, publicado por WitPRESS, Southamptom y Boston, (2012), y el capítulo "Tailandia: Reflejos de la Nueva Reforma Educativa”, El Colegio de México (en proceso).

erangel@ucol.mx 


\section{REFERENCIAS}

Alconchel, I. 2011. "El turismo médico dinamita la salud pública en Tailandia." La Vanguardia, junio 7, 2011. https://www.lavanguardia.com/viajes/20110706/ 54181657307/el-turismo-medico-dinamita-la-sanidad-publica-en-tailandia.html

AP News. 2020. "Asia Today: Thailand lets in some foreigners, open schools." The Associated Press, julio 1, 2020. https://apnews.com/813744896701a8caa3ee9cb637 $\underline{\mathrm{e} 48 \mathrm{f} 35}$

Arunmas, P. 2020a. "US trade move 'won't hurt Thai exports'." Bangkok Post, noviembre 1, 2020. https://www.bangkokpost.com/business/2011779/us-trade-move-wont-hurtthai-exports

Arunmas, P. 2020b. "Business confidence rises in July." Bangkok Post, agosto 21, 2020. https://www.bangkokpost.com/business/1971855/business-confidence-rises-in-july

Bangkok Post. 2020a. "PM urges Asean travel relaunch.” Bangkok Post, junio 27, 2020. https://www.bangkokpost.com/thailand/general/1941628/pm-urges-asean-travelrelaunch

Bangkok Post. 2020b. "50,000 Thai workers to head overseas." Bangkok Post, agosto 17, 2020. https://www.bangkokpost.com/thailand/general/1969259/50-000-thai-workers -to-head-overseas

Bangkok Post. 2020c. "Cambodia to probe activist Wanchalearm's 'abduction'." Bangkok Post, junio 9, 2020. https://www.bangkokpost.com/thailand/general/1931900/cambo dia-to-probe-activist-wanchalearms-abduction

Bangkok Post. 2020d. "Prayut 'not OK' with past handling of 'Boss' Case." Bangkok Post, agosto 6, 2020. https://www.bangkokpost.com/thailand/general/1963879/prayut-notok-with-past-handling-of-boss-case

Bangkok Post, Post Reporters. 2020a. "Suicide rate surges 22\%." Bangkok Post, septiembre 11, 2020. https://www.bangkokpost.com/thailand/general/1983271/suicide-ratesurges-22- 
Bangkok Post, Post Reporters. 2020b. "Khon Kaen locals get former shelter plots.” Bangkok Post, agosto 29, 2020. https://www.bangkokpost.com/thailand/general/1976343/ khon-kaen-locals-get-former-shelter-plots

Beech, Hannah. 2020. "Thai Dissidents are Disappearing, and Families are Fighting for Answers." The New York Times, junio 26, 2020. https://www.nytimes.com/2020/06/ 26/world/asia/thailand-dissidents-disappeared-military.html

Bloomberg. 2020. Misery Index. Bloomberg surveys as of July 27, national statistics agencies. https://www.bloomberg.com/news/newsletters/2020-08-12/supply-chainslatest-soaring-shipping-costs-set-to-go-higher

Brown, Andy. 2020."Thailand turns to transport infrastructure to boost economy, international construction." KHL, agosto 26, 2020. https://www.internationalconstruction.com/news/Thailand-turns-to-transport-infrastructure-to-boosteconomy/1145746.article\#: :text=Thailand's\%20government $\% 20$ is $\% 20$ reported $\% 20 \mathrm{t}$ o,decrease $\% 20 \mathrm{in} \% 20$ exports $\% 20$ and $\% 20$ tourism

Caballero, Noel. 2020. "Una matanza sin precedente en Tailandia se salda con 30 muertos." Agencia EFE, febrero 9, 2020. https://www.efe.com/efe/america/sociedad/unamatanza-sin-precedente-en-tailandia-se-salda-con-30-muertos/20000013-4169421

Camroux, D. 2014. "Duodécimo golpe de Estado en Tailandia." Le Monde diplomatique en español, julio 14, 2014. https://mondiplo.com/duodecimo-golpe-de-estado-en$\underline{\text { tailandia }}$

CISION PR Newswire. 2020. "La nueva cultura del trabajo emerge mientras el mundo cambia." septiembre 7, 2020. https://www.prnewswire.com/es/comunicados-de-prensa/lanueva-cultura-del-trabajo-emerge-mientras-el-mundo-cambia-878785434.html

EFE. 2020. "Las exportaciones de Tailandia caen un $23 \%$ en junio por la pandemia." Investing.com, julio 24, 2020. https://es.investing.com/news/economic-indicators/ las-exportaciones-de-tailandia-caen-un-23--en-junio-por-la-pandemia-2021979

Engblon, Anna, Natthanicha Lephilibert y Nilim Barhua. 2020. "Covid-19: Impact on migrant workers and country response in Thailand." International Labour 
Organization, Country Office for Thailand, Cambodia and Lao PDR, julio 3, 2020. https://www.ilo.org/asia/publications/issue-briefs/WCMS_741920/lang--en/ index.htm

Europapress Internacional. 2020. 'Unas 200,000 personas protestan contra el gobierno de Tailandia y exigen una nueva Constitución.” Europapress Internacional, septiembre 19, 2020. https://www.europapress.es/internacional/noticia-miles-personas-pro testan-contra-gobierno-capital-tailandia-exigen-nueva-constitucion-202009191232 $\underline{05 . h t m l}$

Fan, Jason. 2020. "S'pore \& Thailand to expedite green lane discussions to facilitate essential business travel." Mothership, agosto 26, 2020. https://mothership.sg/2020/08/ singapore-thailand-to-expedite-green-lane-discussions/

Font, Tica y Pere, Ortega. 2012.“Seguridad nacional, seguridad multidimensional, seguridad humana." Papeles de Relaciones Ecosociales y Cambio Global 119: 161-172. https:// www.fuhem.es/papeles_articulo/seguridad-nacional-seguridad-multidimensionalseguridad-humana/

Fry, Gerald Walton. 2018. Education in Thailand: An Old Elephant in Search of a New Mahout. Singapur: UNEVOC. Asia Pacific Educational Research Association/Springer. HEFEI, Xinhua. 2020. “Compañía china inicia con entrega de pedido de trenes a Tailandia.” Xinhua, julio 9, 2020. http://espanol.cri.cn/news/economy/1020/20200907/539531. $\underline{\mathrm{html}}$

Human Rights Watch. 2020. World Report 2020. Events of 2019. https://www.hrw.org/

Japan Today, NEC. 2020. "Orient Link, NEC to build cable system connecting connect Singapore, Malaysia, Myanmar, Thailand and India." Japan Today, agosto 25, 2020.

Karp, Aaron. 2018. “Civilian Firearms Holdings, 2017.” Estimating Global Civilian-Held Firearms Numbers. Geneva: Small Arms Survey. Ginebra: The Graduate Institute of International and Development Studies. 
Kittisilpa, J. 2020. "Thai royalists rally to support monarchy amid calls for reforms." The Straits Times, agosto 30, 2020. https://www.straitstimes.com/asia/se-asia/thai$\underline{\text { royalists-rally-to-support-monarchy-amid-calls-for-reforms }}$

Kunmakara, M. 2020. “Cambodian-Thai trade sees H1 jump, despite pandemic.” The Phnom Penh Post, agosto 24, 2020. https://www.phnompenhpost.com/business/cambodianthai-trade-sees-h1-jump-despite-pandemic

Malay Mail. 2020a. "Govt has helped almost 2,000 Malaysians stranded in Thailand to return home, says ambassador." Malay Mail, agosto 31, 2020. https://www.malaymail. com/news/malaysia/2020/08/31/govt-has-helped-almost-2000-malaysians-strandedin-thailand-to-return-home/1898837

Malay Mail. 2020b. “Lockdown easing boosts Thailand's border trade”. Malay Mail, agosto 17, 2020. https://www.malaymail.com/news/money/2020/08/17/lockdown-easingboosts-thailands-border-trade/1894698

MarketScreener. 2020. "Thailand sees 60\% slump in January-May tourist arrivals, spending." MarketScreener, junio 26, 2020. https://www.marketscreener. com/news/latest/Thailand-sees-60-slump-in-January-May-tourist-arrivals-spending$\underline{-30827791 /}$

National Identity Board. 2000. Thailand into the 2000's. Bangkok: National Identity Board, Office of the Prime Minister, Kingdom of Thailand, 2000.

PNUD. 2019. Informe sobre desarrollo humano 2019. Más allá del ingreso, más allá de los promedios, más allá del presente: Desigualdades del desarrollo humano en el siglo XXI. Nueva York. https://reliefweb.int/sites/reliefweb.int/files/resources/hdr 2019 overview _ _spanish.pdf

Prachatai. 2020. "Protestas juveniles de Tailandia tienen apoyo de ciudadanos que exigen 'democracia real'." Traducido por Romina Navarro. Prachatai, GlobalVoices, septiembre 7, 2020. https://es.globalvoices.org/2020/09/07/protestas-juveniles-detailandia-tienen-apoyo-de-ciudadanos-que-exigen-democracia-real/ 
Rakhmat, M. Z. 2020. "US-China tensions give Southeast Asian nations reason to find other partner elsewhere." The Conversation, agosto 6, 2020. https://theconversation.com/ us-china-tensions-give-southeast-asian-nations-reason-to-find-other-partnerselsewhere-143825

Ramos, Javi. 2020. "El gobierno de Tailandia propone una ley de uniones civiles para parejas del mismo sexo." Dosmanzanas, julio 21, 2020. https://www.dosmanzanas. com/2020/07/el-gobierno-de-tailandia-propone-una-ley-de-uniones-civiles-paraparejas-del-mismo-sexo.html

Reuters. 2020. “Thailand delays China submarines buy amid public outrage.” Reuters, agosto 31, 2020. https://www.reuters.com/article/uk-thailand-china-defence-idUKKBN 25R1JX

Rosales, E. F. 2020. "PHL-Thailand cigarrete dispute still unresolved before WTO." BusinessMirror, julio 1, 2020. https://businessmirror.com.ph/2020/07/01/phlthailand-cigarette-dispute-still-unresolved-before-wto/

Ruiz-Canela, Gaspar. 2020. "Medio millón de apátridas en Tailandia: Sin derecho a tener derechos." Agencia EFE, agosto 3, 2020. https://es-us.noticias.yahoo.com/mediomill\%C3\%B3n-ap\%C3\%A1tridas-tailandia-derecho-153700522.html

Salvá, Ana. 2020. “Más suicidios desde la explosión del coronavirus.” El País, junio 5, 2020. https://elpais.com/elpais/2020/05/22/planeta_futuro/1590156146_603726.html

Shim, E. 2020. “Thailand's royalists decry foreign influence as US issues response." United Press International, septiembre 1, 2020. https:/www.upi.com/Top_News/WorldNews/2020/09/01/Thailands-royalists-decry-foreign-influence-as-US-Embassyissues-response/4761598974656/

Somruedi Banchongduang. 2020. "EIC: 38.4\% more firms shutter in July.” Bangkok Post, septiembre 15, 2020. https://www.bangkokpost.com/business/1985363/eic-38-4more-firms-shutter-in-july 
Spanish.xinhua.com. 2020. "Tailandia cierra temporalmente frontera con Malasia y Myanmar”. Spanish.xinhua.com, marzo 24, 2020. http://spanish.xinhuanet.com/ 2020-03/24/c 138909226.htm

Tanakasempipat, Patpicha y Yanni, Chow. 2020. "Pro-democracy Milk Tea Alliance brews in Asia." Reuters, agosto 18, 2020. https://www.reuters.com/article/us-asiaprotests/pro-democracy-milk-tea-alliance-brews-in-asia-idUSKCN25E102

The ASEAN Post. 2020. "Southeast Asia rapidly ageing population.” The ASEAN Post, febrero 16, 2020. https://theaseanpost.com/article/southeast-asias-rapidly-ageingpopulation\#

The Nation Thailand. 2020a. "Covid-19 takes huge bite out of restaurant giants." The Nation Thailand, agosto 18, 2020. https://www.nationthailand.com/in-focus/30393097

The Nation Thailand. 2020b. "Thailand inks deal with Japan to promote investment, trade." The Nation Thailand, septiembre 1, 2020. https://www.nationthailand.com/in-focus/ $\underline{30393893}$

The Nation Thailand. 2020c. "PM discusses economic recovery with property sectors honchos." The Nation Thailand, septiembre 24, 2020. https://www.nationthailand. com/in-focus/30394006

The Nation Thailand. 2020d. "Cambodian embassy slams door on family of missing Wanchalearm.” The Nation Thailand, agosto 11, 2020. https://www.nationthailand. com/in-focus/30392822

The World Bank. 2020. "Thailand Economic Monitor June 2020: Thailand in the time of Covid-19." International Bank for Reconstruction and Development/The World Bank, junio 30, 2020. https://www.worldbank.org/en/country/thailand/publication/ thailand-economic-monitor-june-2020-thailand-in-the-time-of-covid-19

Tobacco Reporter. 2020. "Philippines-Thailand trade dispute reignites." Tobacco Reporter, agosto 24, 2020. https://tobaccoreporter.com/2020/08/24/philippines-reignites-tradedispute-with-thailand/ 
Transparencia Internacional. 2019. Índice de percepción de corrupción. Berlín. https:/www.transparency.org/es/news/cpi-2019-global-highlights

UNHCR Office. 2020. "Thailand Border Operation RTG/MOI-UNHCR Verified Refugee Population." UNHCR The UN Refugee Agency, agosto 17, 2020. https://reliefweb. int/report/thailand/thailand-border-operation-rtgmoi-unhcr-verified-refugeepopulation-31-july-2020

Vejpongsa, T. 2020. "Students in Thailand protest, call for educational, political reforms." Global News, noviembre 21, 2020. https://globalnews.ca/news/7476652/thai-studentprotests/

VietnamPlus. 2020a. “Tailandia refuerza la lucha antidrogas.” VietnamPlus, agosto 3, 2020. https://es.vietnamplus.vn/tailandia-refuerza-lucha-antidrogas/123700.vnp

VietnamPlus. 2020b. "Repatriados unos 230 vietnamitas desde Tailandia." VietnamPlus, agosto 2, 2020. https://es.vietnamplus.vn/repatriados-unos-230-vietnamitas-desdetailandia/123674.vnp

VietnamPlus. 2020c. "Thailand spends 1.4 billion USD on cash handouts to boost domestic consumption." VietnamPlus, septiembre 3, 2020. https://en.vietnamplus.vn/thailandspends-14-billion-usd-on-cash-handouts-to-boost-domestic-consumption/182354. $\underline{\text { vnp }}$

VietnamPlus. 2020d. "Pandemia de covid-19 impulsa remodelación de industria automotriz de Tailandia." VietnamPlus, julio 20, 2020. https://es.vietnamplus.vn/pandemia-decovid19-impulsa-remodelacion-de-industria-automotriz-de-tailandia/123044.vnp

VietnamPlus. 2020e. "Tailandia aspira a convertirse en primer país de la ASEAN en implementar redes 5G." VietnamPlus, agosto 22, 2020. https://es.vietnamplus.vn/ tailandia-aspira-a-convertirse-en-primer-pais-de-la-asean-en-implementar-redes$\underline{5 \mathrm{~g} / 124533 . v n p}$

VietnamPlus. 2020f. "Thailand works to modernise agriculture with advanced technology." VietnamPlus, septiembre 11, 2020. https://en.vietnamplus.vn/thailand-works-tomodernise-agriculture-with-advanced-technology/182840.vnp 
VietnamPlus. 2020g. "Tailandia enfrenta riesgo de severa escasez de agua, advierten expertos", julio 20, 2020. https://es.vietnamplus.vn/tailandia-enfrenta-riesgo-desevera-escasez-de-agua-advierten-expertos/123079.vnp

WHR. 2020. World Happiness Report. Nueva York. https://worldhappiness.report/ed/2020/

Wongcha-un, Panu y Gabriel Crossley. 2020. "China ready to share water data with Mekong nations, Thailand says.” Reuters, agosto 21, 2020. https://www.reuters.com/ article/us-mekong-river/china-ready-to-share-water-data-with-mekong-nationsthailand-says-idUSKBN25H1JC

Wongcha-un, Panu. 2020. "Thai school \& 'Hunger Games' salute protests spread." Reuters, agosto 18, 2020. https://www.reuters.com/article/us-thailand-protests/thai-schoolhunger-games-salute-protests-spread-idUSKCN25E1AT 